\title{
Sirtuin inhibitors, EX527 and AGK2, suppress cell migration by inhibiting HSF1 protein stability
}

\author{
HYUN-WOO KIM ${ }^{1}$, SOO -A KIM ${ }^{2}$ and SANG-GUN AHN ${ }^{1}$ \\ ${ }^{1}$ Department of Pathology, School of Dentistry, Chosun University, Gwangju 501-759; ${ }^{2}$ Department of Biochemistry, \\ College of Oriental Medicine, Dongguk University, Gyeongju 780-714, Republic of Korea
}

Received July 14, 2015; Accepted September 17, 2015

DOI: $10.3892 /$ or.2015.4381

\begin{abstract}
The histone deacetylases (HDACs), Sirtuin 1 (Sirt1) and Sirt2, play crucial roles in many biological processes, including cell proliferation, differentiation and apoptosis. HDAC inhibitors have been considered as a potential therapeutic approach for various types of cancers. Here, we demonstrated that the Sirt1 and Sirt2 inhibitors EX527 and AGK2 suppressed cell growth and caused G1 phase arrest by inhibiting the expression of Cdk6 and/or Cdk4. An agar colony formation assay revealed that EX527 and AGK2 decreased colony formation in soft agar. Furthermore, EX527 and AGK2 pretreatment inhibited the expression of HSF1 and HSP27 and induced HSF1 ubiquitination. Sirt1 overexpression increased HSF1 expression and/or stabilization and induced cell migration in a scratch assay. Overall, these results indicate that EX527 and AGK2 suppress cell growth and migration by inhibiting HSF1 protein stability.
\end{abstract}

\section{Introduction}

Sirtuins are highly conserved NAD-dependent deacetylases that have been implicated in influencing a wide range of cellular processes, such as aging, transcription, apoptosis, inflammation and stress resistance (1-4). Mammalian sirtuins have seven homologs (Sirt1-7) that share a conserved catalytic domain as class III histone deacetylases (HDACs) $(2,5)$. Of the seven mammalian SIRTs, sirtuin 1 (Sirt1) has been shown to mediate diverse cellular functions, including maintaining genomic stability, suppressing inflammation, enhancing synaptic plasticity, and neuroprotection in models of Alzheimer's disease $(6,7)$. Sirt1 catalyzes the deacetylation of a large number of non-histone substrates, including p53, PGC-1 $\alpha$, NF- $\kappa$ B, PTEN, E2F1 and FOXO transcription factors (8-13). Sirt1 downregulation has been linked to cell senescence and

Correspondence to: Professor Sang-Gun Ahn, Department of Pathology, School of Dentistry, Chosun University, 375 Seosuk-Dong, Dong-gu, Gwangju 501-759, Republic of Korea

E-mail: ahnsg@chosun.ac.kr

Key words: Sirt1, Sirt2, HSF1, HSP27, EX527, AGK2, migration various pathological events such as insulin resistance and severe oxidative stress $(6,9,13,14)$. Like Sirt1, Sirt2 is a strong deacetylase, which deacetylates internal lysines on histone and $\alpha$-tubulin as well as many other proteins such as key transcription factors (15-17). In addition, Sirt2 deacetylates FOXO1 in response to oxidative stress or serum deprivation, thereby negatively regulating FOXO1-mediated autophagy (18). Sirt2 also plays a major role in cell cycle progression and genomic stability $(19,20)$. A recent study showed that Sirtl deacetylates HSF1 and potentiates its DNA-binding ability, and the downregulation of Sirt1 accelerates the release of HSF1 from its cognate promoter elements and attenuates the heat shock response $(21,22)$. Another study showed that the Sirt1 activator SRT1720 prevents colitis by reducing HSF1 acetylation and increasing the expression of BIP, HSP27 and HSP90. Sirt1 activation thus participates in protecting cells from stresses related to damaged, misfolded or aggregated proteins (23). However, the mechanism by which Sirt1 and Sirt 2 may regulate HSF1 is still unclear.

We were interested in gaining a better understanding of the role of Sirt1 and Sirt2 as regulators of HSF1. We found that inhibitors of Sirt1 and Sirt2, EX527 and AGK2, induced the acetylation and the degradation of heat stress-induced HSF1. In addition, cellular HSF1 stability, by controlling Sirt1 expression, modulated cell migration by influencing HSP27 expression.

\section{Materials and methods}

Cell culture and reagents. HeLa cells were cultured in Minimal Essential Medium (MEM; Sigma-Aldrich, St. Louis, MO, USA) supplemented with 10\% FBS (Biochrom AG, Berlin, Germany), $100 \mathrm{U} / \mathrm{ml}$ penicillin and $100 \mu \mathrm{g} / \mathrm{ml}$ streptomycin (Sigma-Aldrich) and maintained in $5 \% \mathrm{CO}_{2}$ at $37^{\circ} \mathrm{C}$. EX527, AGK2 and hemin were purchased from Sigma-Aldrich. Fugene HD transfection reagent was from Promega (Madison, WI, USA). Polyclonal anti-HSF1, anti-HSP27, anti-caspase-3, and anti-PARP antibodies were from Santa Cruz Biotechnology, Inc. (Dallas, TX, USA). Monoclonal anti-Sirt1 and anti-Sirt2 antibodies were from Cell Signaling Technology (Danvers, MA, USA).

Reverse transcription-polymerase chain reaction. Total RNA of HeLa cells was extracted using TRIzol reagent (Life 
Technologies, Gaithersburg, MD, USA) according to the manufacturer's instructions. A total of $1 \mu \mathrm{g}$ of total RNA was reverse-transcribed to cDNA in a reaction mixture of $20 \mu \mathrm{l}$ with the Reverse Transcription system (Promega, Leiden, The Netherlands). Amplification was performed for 30 cycles in a DNA thermal cycler. GAPDH was used as a control for the PCR reaction. The PCR products were resolved on a $1.5 \%$ agarose gel and were stained with ethidium bromide.

Cell proliferation assay. Cell proliferation was assessed using a 3-(4,5-dimethylthiazolyl-2)-2,5-diphenyltetrazolium bromide (MTT) assay. The cells were seeded in a 96-well plate at a density of $1 \times 10^{4}$ cells/well. The next day, the cells were washed twice with PBS, and $500 \mu \mathrm{g} / \mathrm{ml}$ MTT (Sigma-Aldrich) was added to the wells. The MTT solution was removed after $4 \mathrm{~h}$ of incubation at $37^{\circ} \mathrm{C}$. A mixture of $0.01 \mathrm{M}$ glycine and DMSO (Sigma-Aldrich) was added to each well. The absorbance was measured at $540 \mathrm{~nm}$ with a Benchmark microplate reader (Bio-Rad Laboratories, Philadelphia, PA, USA).

Immunoprecipitation. Total cell extracts were incubated with anti-HSF1 in NP-40 lysis buffer (0.5\% NP-40, $0.5 \%$ Triton $\mathrm{X}-100,150 \mathrm{mM} \mathrm{NaCl}, 50 \mathrm{mM}$ Tris $\mathrm{HCl} \mathrm{pH}$ 7.4, 1 mM EDTA, $50 \mathrm{mM} \mathrm{NaF}, 1 \mathrm{mM}$ B-glycerophosphate, $1 \mathrm{mM}$ sodium orthovanadate, $0.5 \mu \mathrm{g} / \mathrm{ml}$ leupeptin, $1 \mu \mathrm{g} / \mathrm{ml}$ pepstatin, $0.2 \mathrm{mM}$ PMSF). The extract mixtures were incubated at $4^{\circ} \mathrm{C}$ overnight with rotation before the addition of $20 \mu \mathrm{l}$ of protein $\mathrm{A} / \mathrm{G}$ beads (Life Technologies) for $3 \mathrm{~h}$ at $4^{\circ} \mathrm{C}$. The beads were washed three times with the same buffer and suspended in 2X SDS sample buffer. The samples were resolved in SDS-polyacrylamide gels for western blot analysis with specific antibodies as indicated.

Western blot analysis. Cell lysates $(50 \mu \mathrm{g})$ were placed in lysis buffer (50 mM Tris-HCl, pH 7.5, $150 \mathrm{mM} \mathrm{NaCl}, 1 \%$ NP-40, $0.5 \%$ Na-deoxycholate, $0.1 \%$ SDS, and a protease inhibitor cocktail containing $1 \mu \mathrm{g} / \mathrm{ml}$ aprotinin and leupeptin) and separated by $12 \%$ SDS-PAGE. The resolved proteins were transferred onto a nitrocellulose membrane (Amersham Pharmacia Biotech, UK) according to standard procedures. The membrane was blocked in 5\% non-fat dry milk for $3 \mathrm{~h}$ and incubated with the primary antibodies for $3 \mathrm{~h}$ at room temperature (RT). After incubation with a specific peroxidase-coupled secondary antibody (Sigma-Aldrich) for $1 \mathrm{~h}$, the blotted bands were detected using an enhanced chemiluminescence detection kit (Amersham Pharmacia Biotech).

Wound-scratch assays. Cells were allowed to grow in a culture dish overnight, and a scratch $\sim 3$-mm wide was created in the monolayer using a pipette tip. After washing twice with PBS, the cells were treated with or without the Sirt1/Sirt2 inhibitors, and images were captured after 12 or $36 \mathrm{~h}$. Cells were imaged in 5 random microscopic fields per well using an Olympus IX2-SLP inverted microscope (Japan) at a X100 magnification.

Annexin V-FITC/PI double staining. The cells were harvested and fixed with $70 \%$ ethanol for $1 \mathrm{~h}$ at $4^{\circ} \mathrm{C}$ for cell cycle analysis. After washing with cold PBS, the cells were incubated with DNase-free RNase and propidium iodide (PI) at $37^{\circ} \mathrm{C}$ for $30 \mathrm{~min}$. The specific binding of Annexin V-FITC/PI was performed by incubating the cells for $15 \mathrm{~min}$ at RT in a binding buffer (10 mM HEPES, $\left.140 \mathrm{mM} \mathrm{NaCl}, 2.5 \mathrm{mM} \mathrm{CaCl}_{2}, \mathrm{pH} 7.4\right)$ containing saturated concentrations of Annexin V-FITC and PI. After incubation, the cells were pelleted and analyzed in a FACScan analyzer (Beckman Coulter Inc., Fullerton, CA, USA).

Flow cytometry and cell cycle analysis. The cell cycle distribution was analyzed by flow cytometry. Briefly, $1 \times 10^{6}$ cells were harvested and washed in PBS, and then fixed in $70 \%$ alcohol for $30 \mathrm{~min}$ at $4^{\circ} \mathrm{C}$. After washing three times in cold PBS, the cells were resuspended in $1 \mathrm{ml}$ of PBS solution containing $50 \mu 1$ of $1 \mathrm{mg} / \mathrm{ml} \mathrm{PI}$ and 1 unit of DNase-free RNase for $30 \mathrm{~min}$ at $37^{\circ} \mathrm{C}$. The samples were then analyzed for their DNA content by FACS (Beckman Coulter Inc.).

Soft agar colony formation assay. Briefly, the cells $\left(8 \times 10^{3}\right.$ cells/well) were exposed to different concentrations of EX527 or AGK2 in $1 \mathrm{ml}$ of $0.3 \%$ basal medium Eagle's agar containing $10 \%$ FBS. The cultures were maintained at $37^{\circ} \mathrm{C}$ in a $5 \% \mathrm{CO}_{2}$ incubator for 10-15 days, and the cell colonies were scored using an Olympus IX2-SLP inverted microscope (Japan).

Statistical analyses. All experiments were performed at least three times. The mean values for the experiments are expressed as the mean \pm SE. Significant differences were assessed by analysis of variance. $\mathrm{p}<0.05$ was considered to indicate a statistically significant difference.

\section{Results}

Cytotoxic effect of the Sirt1/2 inhibitors EX527 and AGK2 on cell proliferation. To examine the effect of Sirt1/2 inhibitors, EX527 and AGK2, on the growth of HeLa cells, we performed MTT assays. The cells were exposed to increasing concentrations of nicotinamide (NAM), EX527 and AGK2 for $24 \mathrm{~h}$, and the cell viability was monitored (Fig. 1). EX527 and AGK2 significantly inhibited cell proliferation in a dose-dependent manner. EX527 at concentrations of 50 and $100 \mu \mathrm{M}$ resulted in cell growth inhibition of 68 and 54\%, respectively, at $24 \mathrm{~h}$ (Fig. 1B). Similarly, AGK2 also significantly inhibited cell growth in a dose-dependent manner without inducing cytotoxicity at low doses ( $\geq 1 \mu \mathrm{M}$; Fig. 1C). However, these cells exhibited no significant decrease in cell proliferation after NAM treatment for $24 \mathrm{~h}$ (survival rate $>93 \%$; Fig. 1A).

To further assess the effect of EX527 and AGK2 on tumorigenicity, we compared the anchorage-independent growth rates. Twelve days after EX527 $(50 \mu \mathrm{M})$ and AGK2 $(5 \mu \mathrm{M})$ treatment, HeLa cells showed a significantly reduced colony forming ability in soft agar to $~ 95$ and $46 \%$ of the control cells, respectively (Fig. 1D). Together, our observations showed that EX527 and AGK2 suppressed the malignant phenotype such as cell proliferation and colony formation.

EX527 and AGK2 induce G1 cell cycle arrest in HeLa cells. It was hypothesized that EX527 or AGK2 induce alterations in cell cycle regulation. Using flow cytometry, we analyzed the effect of EX527 or AGK2 on cell cycle progression. As shown in Fig. 2A-C, EX527 and AGK2 induced cell cycle arrest at the G1 phase. Western blot analysis showed that the levels of CDK4 or CDK6 and cyclin D1 were decreased after EX527 or AGK2 
A

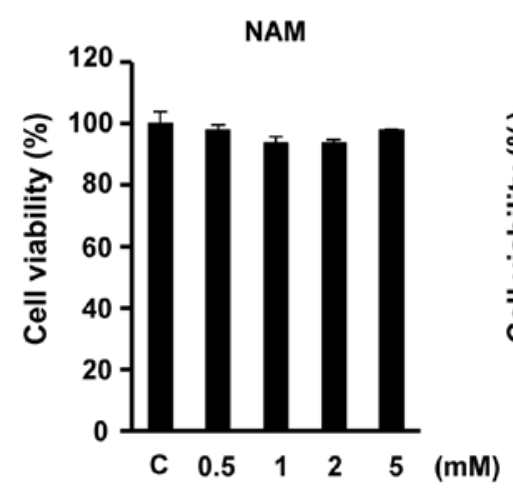

B

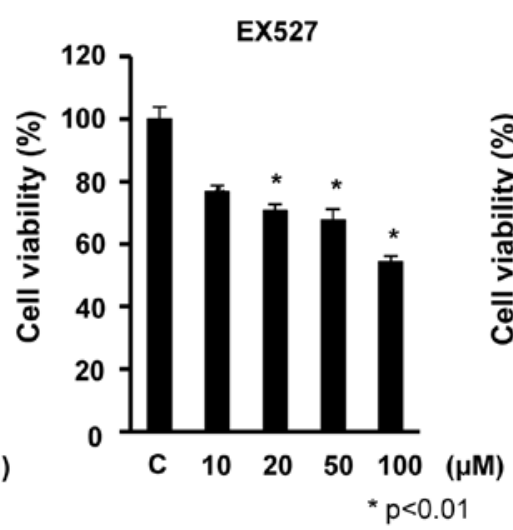

C

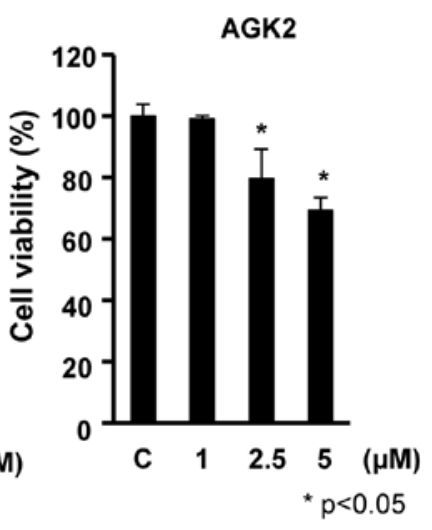

D
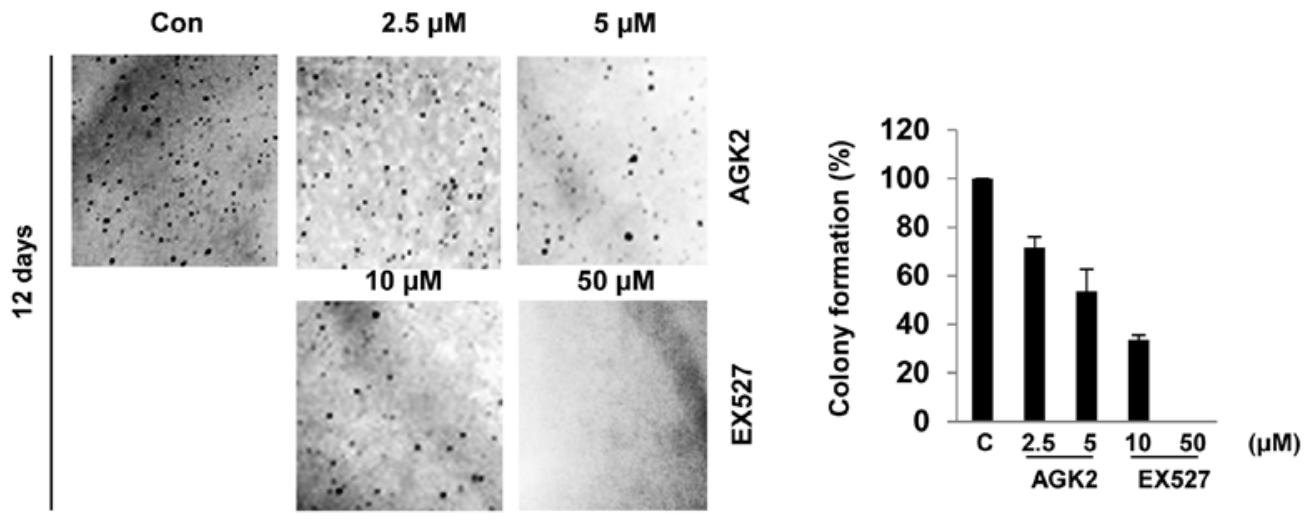

Figure 1. The effects of EX527 and AGK2 on cell proliferation. HeLa cells were exposed to different concentrations of NAM (A), EX527 (B) and AGK2 (C) for $24 \mathrm{~h}$. Cell viability was assessed using an MTT assay. (D) EX527 and AGK2 inhibited cell colony formation on soft agar. Cell suspensions were mixed with $0.3 \%$ agar in the culture medium with EX527 and AGK2. After 12 days of incubation at $37^{\circ} \mathrm{C}$ in a $5 \% \mathrm{CO}_{2}$ incubator, the colonies were counted. Representative phase contrast images are shown. The data represent the average number of colonies per plate as determined from three separate experiments.

treatment in a dose-dependent manner. In addition, EX527 and AGK 2 inhibited the expression of p53 protein (Fig. 2D). These results suggest that the effects of EX527 and AGK2 on G1 cell cycle progression were associated with the inhibition of cell growth.

To determine whether EX527 and AGK2 induce death in HeLa cells, we quantified apoptosis by flow cytometry using the Annexin V-FITC/PI double staining assay. As shown in Fig. 2E-H, after $24 \mathrm{~h}$ of incubation, there was no significant decrease in living cells upon EX527 treatment; 93\% of the control cells were alive, while only $3.3 \%$ of the EX527-treated cells underwent cell death. Similarly, apoptotic cells were not markedly observed after AGK2 treatment (Fig. 2G). Consistent with this observation, caspase-3 and PARP were not cleaved in cells treated with EX527 or AGK2 compared with the control (Fig. 2H). In addition, key autophagy proteins, LC3B and beclin-1, were not activated after treatment with EX527 or AGK2 (Fig. 2I).

EX527 and AGK2 inhibit the HSF1/HSP27 pathway. A previous study suggested that Sirt1 deacetylates HSF1 and activates heat shock proteins (HSPs) under a heat stress condition (21). We investigated the possibility that Sirt1 and Sirt2 inhibition by EX527 and AGK2 may be responsible for HSF1 inactivation. Therefore, we subjected EX527- or AGK2-treated cells to heat shock $\left(1 \mathrm{~h}\right.$ at $\left.42^{\circ} \mathrm{C}\right)$ and analyzed the expression of endogenous Sirt1, Sirt2 and HSF1, as well as HSPs such as Hsp27 and Hsp70. EX527 or AGK2 treatment decreased the expression of HSF1 and HSP27 in non-stress or heat stress condition (Fig. 3A and B), which indicated that Sirt1 and Sirt 2 are required for the regulation of the HSF1 pathway. Furthermore, EX527 and AGK2 also decreased HSF1 phosphorylation after heat shock. However, NAM decreased HSF1 expression/phosphorylation and HSP27 expression under heat shock conditions (Fig. 3C).

To determine whether EX527 and AGK2 influence HSF1 transcription in HeLa cells, RT-PCR was performed using specific oligonucleotides against the HSF1 gene. As shown in Fig. 3D, the HSF1 mRNA level was not altered by EX527 or AGK2.

To verify the significance of the regulation of HSF1 by Sirt1, cells were transfected with or without Sirt1, exposed to a $42^{\circ} \mathrm{C}$ heat shock for $1 \mathrm{~h}$, allowed to recover at $37^{\circ} \mathrm{C}$ for $24 \mathrm{~h}$, and analyzed for HSF1 expression. As expected, the heat shock induced HSP27 expression, which increased with recovery time. Importantly, at the same time-points, we observed that the cells overexpressing Sirtl showed increased HSF1 expression compared with the heat shock-treated cells (Fig. 3E). 
A

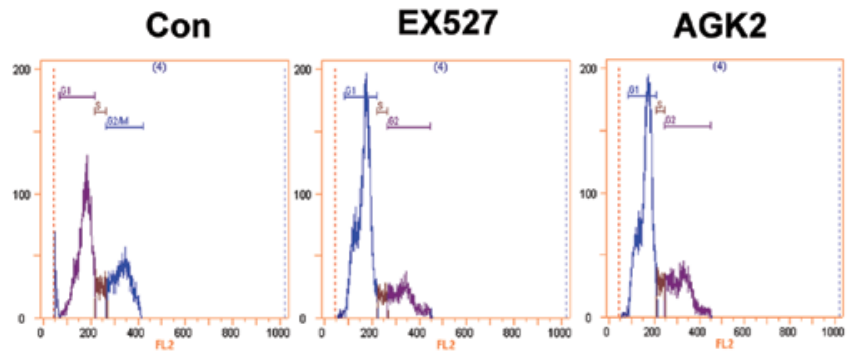

B

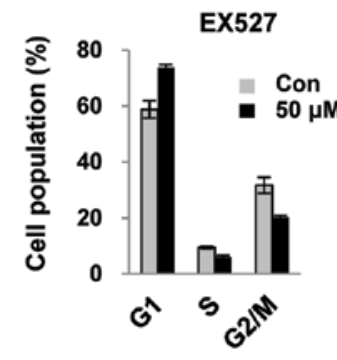

C

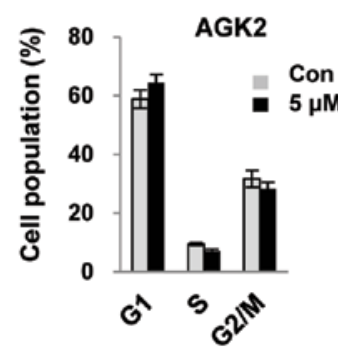

D

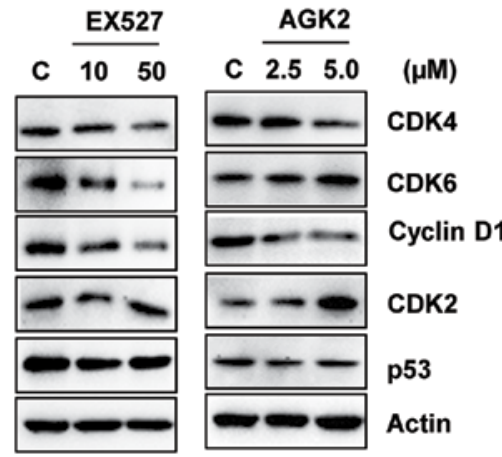

E

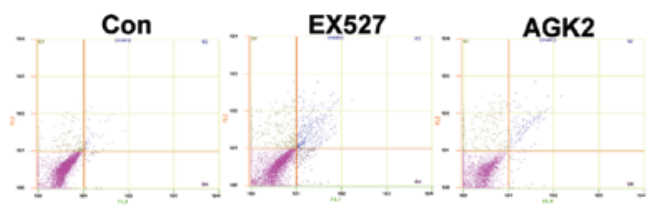

$\mathbf{F}$

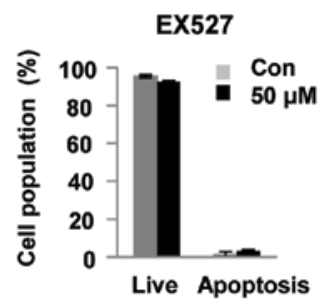

G

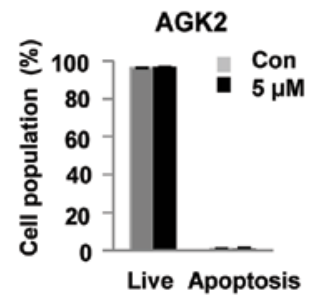

H

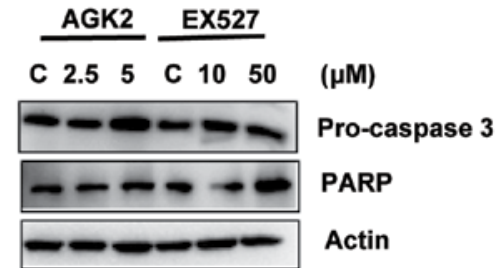

I

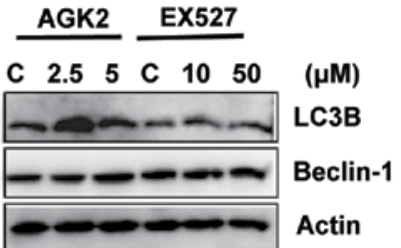

Figure 2. EX527 or AGK2 leads to G1 phase arrest through CDK4/6 and cyclin D1 downregulation. (A) Cells were exposed to EX527 (10 or $50 \mu \mathrm{M})$ or AGK2 $(2.5$ or $5 \mu \mathrm{M}$ ) for $24 \mathrm{~h}$ and then subjected to flow cytometric analysis of propidium iodide (PI)-stained cells. (B and C) The percentage of cells in the phases of the cell cycle was determined. (D) Protein expression levels were determined by western blotting. (E-G) Cell death was determined by flow cytometric analysis of Annexin V/PI double staining. (H and I) Representative immunoblotting for caspase-3, PARP, LC3B, and beclin-1. The cells were exposed to EX527 or AGK2 for $24 \mathrm{~h}$.

Sirtl/Sirt2 inhibition is linked to increased HSF1 ubiquitination. To determine whether HSF1 deacetylation by Sirt1 is involved in HSF1 ubiquitination/stabilization, we examined the acetylation and ubiquitination status of HSF1 after EX527 and AGK2 treatment. HeLa cells were treated with or without EX527 and AGK2 and exposed to a $42^{\circ} \mathrm{C}$ heat shock for $1 \mathrm{~h}$. Then, the samples were immunoprecipitated with an anti-HSF1 antibody and analyzed for HSF1 acetylation. Acetylated HSF1 was detected in the untreated cells but was decreased in the cells exposed to heat shock stress conditions. However, acetylated HSF1 levels were induced in the EX527or AGK2-treated cells before heat shock (Fig. 4A). In addition, EX527 and AGK2 induced HSF1 ubiquitination (Fig. 4B).

To investigate whether proteasome-dependent protein degradation is involved in Sirtl inhibitor-mediated downregulation of HSF1 protein, we treated cells with the proteasome inhibitor hemin and/or EX527. As shown in Fig. 4C and D, the HSF1 protein level in the hemin-treated cells was substantially higher than that in the EX526-treated cells. Our above data indicate that EX527 or AGK2 decreases the expression/ phosphorylation of HSF1 protein. However, hemin inhibited the EX527-mediated reduction of the HSF1 protein. From the above data, we conclude that Sirt1 and/or Sirt2 inhibition results in a diminution of $\mathrm{HSF} 1$ protein stabilization.

EX527 and AGK2 inhibit cell migration. To investigate whether Sirt1/HSF1 modulate cell motility, we treated HeLa cells with EX527 and AGK2 for $36 \mathrm{~h}$ and performed a wound-healing experiment. EX527- or AGK2-treated cells showed reduced migration ability when compared with the control or heat shock-treated cells (Fig. 5A).

To further assess the effect of the Sirt1/HSF1/HSP27 pathway on cell migration, we compared the migration capabilities of Sirt1 or HSF1 with or without siHSP27 
A

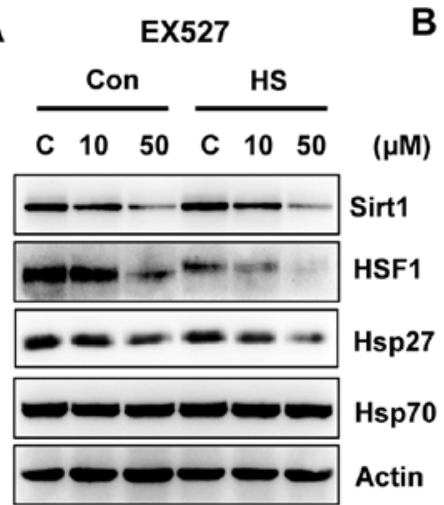

D

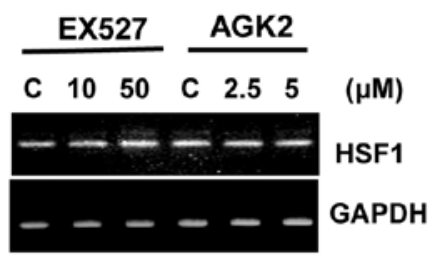

B

E
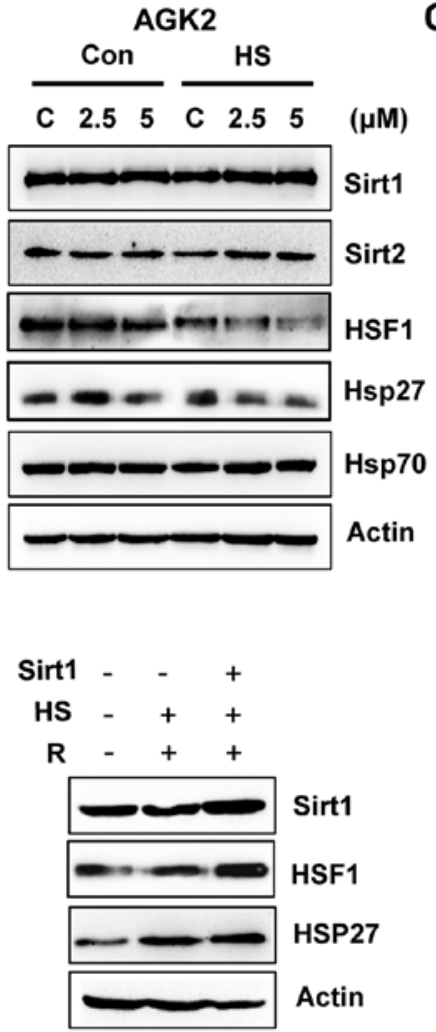

C

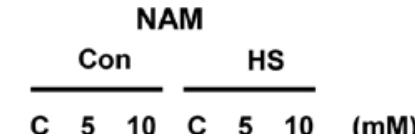

$\begin{array}{llllllll}C & 5 & 10 & C & 5 & 10 & (\mathrm{mM})\end{array}$

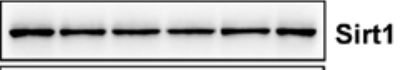

$----\infty$ Sirt2
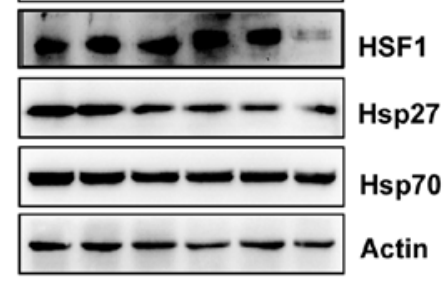

Figure 3. EX527 and AGK2 inhibit heat shock-induced HSF1 expression. HeLa cells were treated with EX527 (A), AGK2 (B), or NAM (C) for 24 h with or without heat shock treatment (HS) $\left(42^{\circ} \mathrm{C}\right.$ for $1 \mathrm{~h}$ ). Cells were then lysed, and the levels of Sirt1/2, HSF1, HSP27, and HSP70 protein were detected by western blotting. (D) HSF1 mRNA levels were determined by RT-PCR in EX527- and AGK2-treated cells. (E) HeLa cells were transfected with or without pCMV5-Sirt1 for $48 \mathrm{~h}$, exposed to a $42^{\circ} \mathrm{C}$-heat shock for $1 \mathrm{~h}$ and were allowed to recover at $37^{\circ} \mathrm{C}$ for $24 \mathrm{~h}$. R: $37^{\circ} \mathrm{C}$ recovery after heat shock.

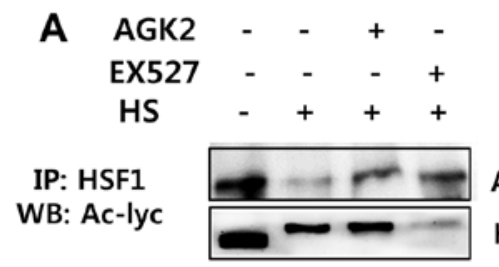

B

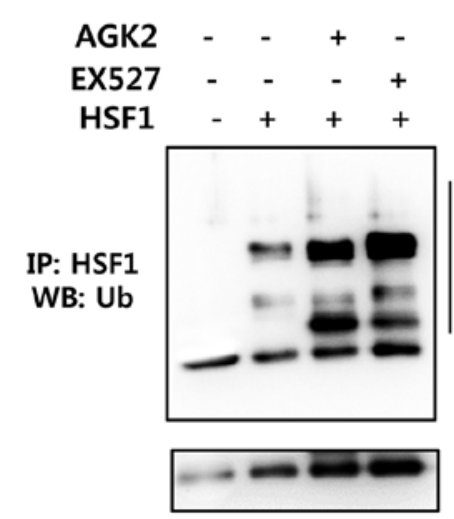

C

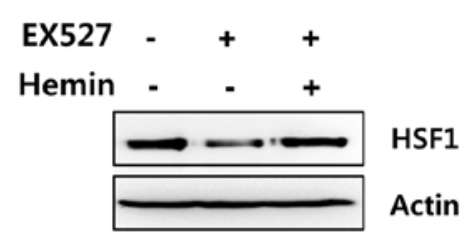

D

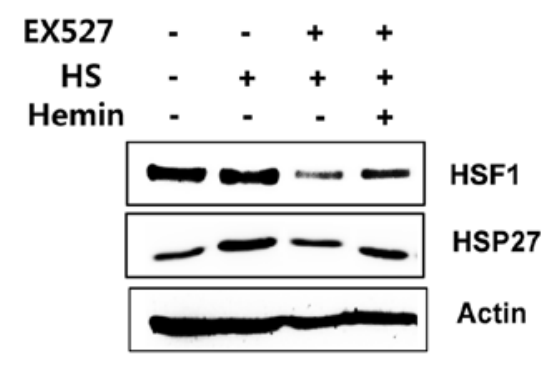

HSF1

Figure 4. The effect of EX527 and AGK2 on HSF1 ubiquitination. (A) Acetylation status of HSF1 was analyzed after HeLa cells treated with EX527 or AGK2 were subjected to immunoprecipitation with anti-acetyl antibodies. (B) EX527 and AGK2 induce HSF1 ubiquitination. Cells were transfected with an HSF1 expression vector. Twenty-four hours after transfection, the cells were treated with EX527 or AGK2 for 24 h. Cell lysates were obtained and subjected to immunoprecipitation using an anti-HSF1 antibody followed by western blot analysis using an anti-ubiquitin antibody (upper panel). Hsp70 levels in the cells were monitored by western blot analysis with an anti-HSF1 antibody (lower panel). (C and D) EX527 accelerates proteasome-mediated degradation of HSF1. EX527treated cells were treated with $30 \mu \mathrm{M}$ hemin with or without heat shock stress (HS) for $24 \mathrm{~h}$, and western blot analysis was performed for HSF1 and HSP27.

transfection. First, the levels of the HSF1 protein increased in the HSF1-transfected cells compared to the control cells (Fig. 5B). As expected, HSF1-overexpression led to induced Sirtl protein levels. A wound-healing assay showed 
A

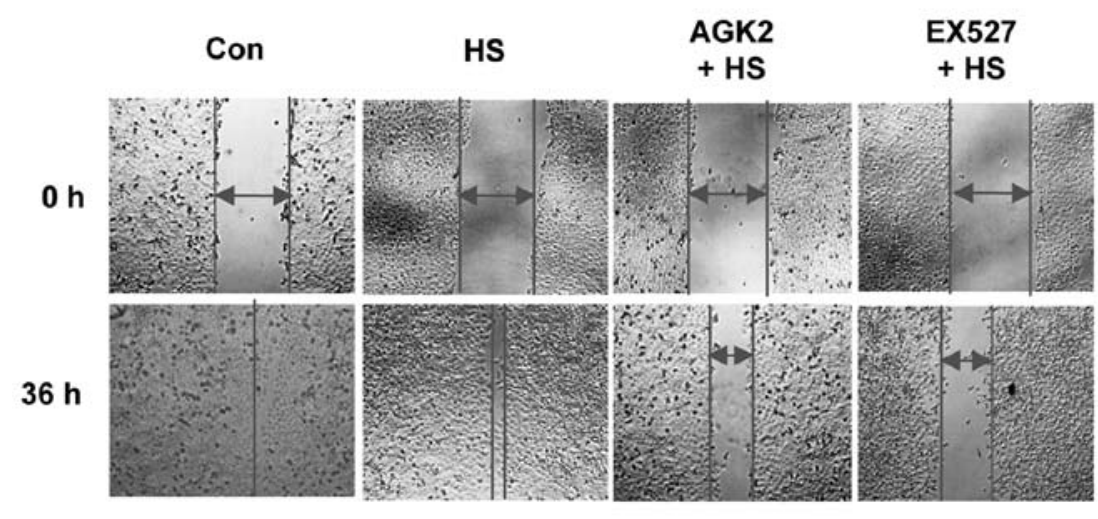

B

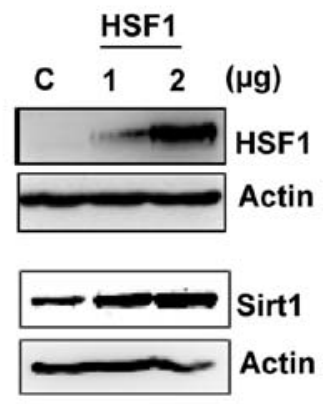

\section{C}

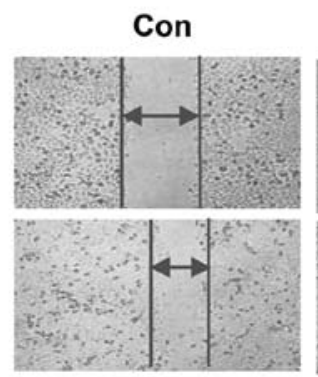

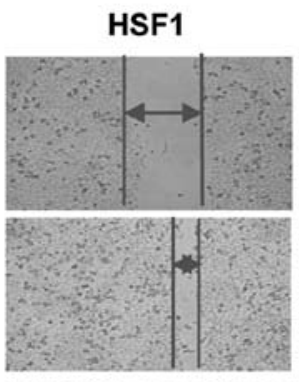

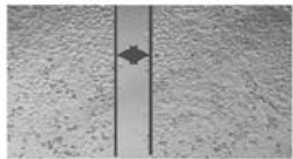

HSF1 + siHSP27

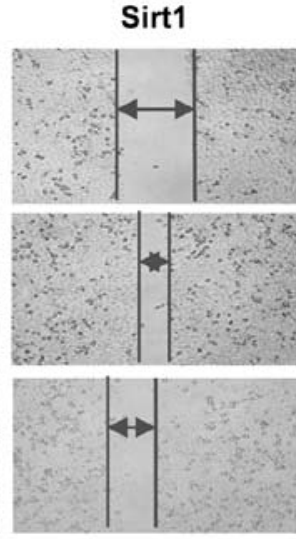

Sirt1 + siHSP27

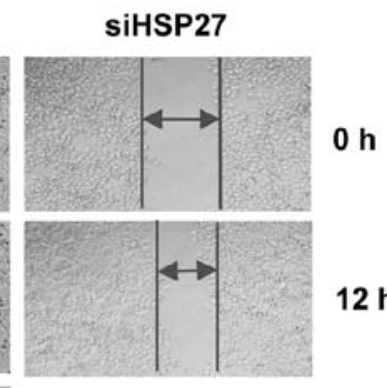

$12 \mathrm{~h}$

Figure 5. Cell migration is regulated by the Sirt1/2-mediated HSF1/HSP27 pathway. (A) A wound-healing assay shows the closure rates of cells treated with EX527 and AGK2. (B and C) The effects of HSF1, Sirt1, and siHSP27 on cell migration. Cells were transfected with HSF1 or Sirt1 for $48 \mathrm{~h}$ and subjected to western blotting. A wound-healing assay shows the closure rates of cells transfected with HSF1, Sirt1, and/or siHSP27. Images of wound-closure rates at the indicated time-points.

that overexpression of HSF1 or Sirt1 led to increased cell migration. However, the increase in gap closure was delayed in cells transfected with siHSP27; the migration of siHSP27transfected cells was similar to the control cells (Fig. 5C). These data indicate that Sirt1/Sirt2-mediated HSF1/HSP27 regulation is involved in cell migration.

\section{Discussion}

In the present study, the pharmacological inhibition of Sirt1 or Sirt2 by the specific inhibitor EX527 or AGK2 caused G1 phase arrest, which was mediated by the inhibition of Cdk4, Cdk6, and cyclin D1 and reduced the cell growth and colony formation of HeLa cells. Although EX527- or AGK2-treated HeLa cells exhibited decreased cell growth due to cell cycle arrest at the G1 phase, they did not exhibit higher levels of apoptosis or autophagy.

Previous studies have suggested that other Sirt inhibitors, such as AC-93253 and AEM1/2, reduce cell proliferation by inducing apoptosis (23-26). Other reports have linked Sirt1 to the regulation of autophagy, such as the report that showed that Sirt1 (-/-) mouse embryonic fibroblasts fail to activate autophagy in response to nutrient deprivation (27). The mechanisms underlying the effect of Sirt1/2 on cell death remain to be determined but may be dependent on cell type and the duration of Sirt1/2 inhibitor treatment.

Our study revealed that EX527 and AGK2 negatively regulated the expression and/or phosphorylation of HSF1, but the mRNA level of HSF1 did not change. In addition, reduced HSF1 by EX527 or AGK2 was recovered in the presence of hemin, a proteasome inhibitor, suggesting that HSF1 may be degraded by proteasomal machinery following EX527 or AGK2 treatment. To confirm this hypothesis, we observed that the inhibition of HSF1 deacetylation by EX527 and AGK2 reduced HSF1 protein abundance through HSF1 ubiquitination, indicating that the ubiquitin-proteasome system exerts an important role in the acetylation/deacetylation status of the HSF1 protein. The additional effect of HSF1 acetylation could induce HSF1 degradation in the nucleus and inhibit target genes involved in heat shock response, such as HSP27.

Previous studies have shown that Sirt1 is recruited to the hsp70 promoter and activates HSF1 through deacetylation at a key lysine 80 residue within the DNA binding domain. In contrast, Sirt1 downregulation accelerates the release of HSF1 from its cognate promoter elements and attenuates the heat shock response (HSR) (21-23). Additionally, Sirtl was shown to deacetylate HSF1 and increase HSP70 levels in the brains of A57T mice, leading to $\alpha$-synuclein aggregate suppression (28). 
Consistently, Sirt1/2 inhibition with EX527 and AGK2 inhibited HSF1-mediated HSP27 induction under heat shock conditions. These findings could be explained as follows. First, Sirt1/2 deacetylates HSF1 by binding to HSF1 and promotes the heat shock response. Second, in contrast, inhibition of Sirt1/2 accelerates HSF1 ubiquitination likely by acetylating the lysine residue and ubiquitinated HSF1 is recognized and degraded by the proteasome system.

During elevated stress, HSF1 is subject to additional enzymatic post-translational modifications including phosphorylation and SUMOylation as well as acetylation $(21,29,30)$. These modifications are thought to promote HSF1 expression/activity to respond to various stresses. The acetylation/deacetylation status of HSF1 may affect several molecular pathways involved in the regulation of cell protection, migration/movement, and stress resistance including MAP kinase signaling, vinculin, and heat shock protein 27 (Hsp27) (31-33).

As described above, our findings suggest that the expression/ activation of HSF1/HSP27 depend at least in part on the Sirt1/ Sirt2 pathway. This finding prompted us to investigate whether the Sirt1/2-regulated HSF1/HSP27 pathway may affect motility such as migration. We demonstrated that the inhibition of Sirt1/2 by EX527 or AGK2 decreased cell motility in a scratch assay. Our data indicate that Sirt1 and HSF1/HSP27 play an important role in cell migration, as specifically demonstrated by the findings that overexpression of Sirt1 and HSF1 increased cell migration, whereas downregulation of HSP27 by siRNA decreased Sirt1- and HSF1-induced cell migration. The direct causal association between the HSF1/HSP27 pathway and Sirt1/2 was apparent by the finding that modulation of Sirt1/2 activity by EX527 and AGK2 strongly affected HSF1 expression/activation and modulated cell migration. The molecular basis for the activity of HSF1/HSP27 in affecting cell motility and in modulating the response to Sirt1 and Sirt2 still needs to be clarified. It should also be considered that, although Sirt1 is involved in HSF1 deacetylation, additional deacetylation sites by Sirt 2 on HSF1 activation are present, which could be differentially regulated by Sirt1 and Sirt 2 and may modulate other cellular functions in these cells.

In conclusion, the inhibition of Sirt1/2 by EX527 or AGK2 inhibited cell growth and colony formation. Our study is the first to demonstrate that blocking Sirt1 and Sirt2 can induce HSF1 ubiquitination and degradation in vitro, suggesting that Sirt1/2 is involved in the activation of heat shock response signaling pathways. Furthermore, we observed a consistent reduction in cell migration after EX527 and AGK2 treatment. Our results suggest that Sirt1 and Sirt2 regulate cell migration via HSF1/Hsp27-mediated signaling.

\section{Acknowledgements}

This study was supported by a National Research Foundation of Korea (NRF) grant funded by the Korean government MSIP (no. 2008-0062283).

\section{References}

1. Alcendor RR, Gao S, Zhai P, Zablocki D, Holle E, Yu X, Tian B, Wagner T, Vatner SF and Sadoshima J: Sirtl regulates aging and resistance to oxidative stress in the heart. Circ Res 100: $1512-1521,2007$.
2. Michan S and Sinclair D: Sirtuins in mammals: Insights into their biological function. Biochem J 404: 1-13, 2007.

3. Finkel T, Deng CX and Mostoslavsky R: Recent progress in the biology and physiology of sirtuins. Nature 460: 587-591, 2009.

4. Yang T and Sauve AA: NAD metabolism and sirtuins: Metabolic regulation of protein deacetylation in stress and toxicity. AAPS J 8: E632-E643, 2006.

5. Pang M and Zhuang S: Histone deacetylase: A potential therapeutic target for fibrotic disorders. J Pharmacol Exp Ther 335: 266-272, 2010.

6. Kwon HS and Ott M: The ups and downs of SIRT1. Trends Biochem Sci 33: 517-525, 2008.

7. Lavu S, Boss O, Elliott PJ and Lambert PD: Sirtuins - novel therapeutic targets to treat age-associated diseases. Nat Rev Drug Discov 7: 841-853, 2008.

8. Luo J, Nikolaev AY, Imai S, Chen D, Su F, Shiloh A, Guarente L and $\mathrm{Gu}$ W: Negative control of p53 by Sir2alpha promotes cell survival under stress. Cell 107: 137-148, 2001.

9. Garcia MM, Guéant-Rodriguez RM, Pooya S, Brachet P, Alberto JM, Jeannesson E, Maskali F, Gueguen N, Marie PY, Lacolley P, et al: Methyl donor deficiency induces cardiomyopathy through altered methylation/acetylation of PGC-1 $\alpha$ by PRMT1 and SIRT1. J Pathol 225: 324-335, 2011.

10. Yeung F, Hoberg JE, Ramsey CS, Keller MD, Jones DR, Frye RA and Mayo MW: Modulation of NF-kappaB-dependent transcription and cell survival by the SIRT1 deacetylase. EMBO J 23: 2369-2380, 2004.

11. Ikenoue T, Inoki K, Zhao B and Guan KL: PTEN acetylation modulates its interaction with PDZ domain. Cancer Res 68: 6908-6912, 2008.

12. Wang C, Chen L, Hou X, Li Z, Kabra N, Ma Y, Nemoto S, Finkel T, Gu W, Cress WD, et al: Interactions between E2F1 and SirT1 regulate apoptotic response to DNA damage. Nat Cell Biol 8: 1025-1031, 2006

13. Brunet A, Sweeney LB, Sturgill JF, Chua KF, Greer PL, Lin Y, Tran H, Ross SE, Mostoslavsky R, Cohen HY, et al: Stress-dependent regulation of FOXO transcription factors by the SIRT1 deacetylase. Science 303: 2011-2015, 2004.

14. Zhao G, Cui J, Zhang JG, Qin Q, Chen Q, Yin T, Deng SC, Liu Y, Liu L, Wang B, et al: SIRT1 RNAi knockdown induces apoptosis and senescence, inhibits invasion and enhances chemosensitivity in pancreatic cancer cells. Gene Ther 18: 920-928, 2011.

15. SkogeRH,Dölle C andZiegler M:Regulation of SIRT2-dependent $\alpha$-tubulin deacetylation by cellular NAD levels. DNA Repair (Amst) 23: 33-38, 2014.

16. Patel VP and Chu CT: Decreased SIRT2 activity leads to altered microtubule dynamics in oxidatively-stressed neuronal cells: Implications for Parkinson's disease. Exp Neurol 257: 170-181, 2014.

17. Donmez G and Outeiro TF: SIRT1 and SIRT2: Emerging targets in neurodegeneration. EMBO Mol Med 5: 344-352, 2013.

18. Wang F, Nguyen M, Qin FX and Tong Q: SIRT2 deacetylates FOXO3a in response to oxidative stress and caloric restriction. Aging Cell 6: 505-514, 2007.

19. Serrano L, Martínez-Redondo P, Marazuela-Duque A, Vazquez BN, Dooley SJ, Voigt P, Beck DB, Kane-Goldsmith N, Tong Q, Rabanal RM, et al: The tumor suppressor SirT2 regulates cell cycle progression and genome stability by modulating the mitotic deposition of H4K20 methylation. Genes Dev 27: 639-653, 2013.

20. Kim HS, Vassilopoulos A, Wang RH, Lahusen T, Xiao Z, Xu X, Li C, Veenstra TD, Li B, Yu H, et al: SIRT2 maintains genome integrity and suppresses tumorigenesis through regulating APC/C activity. Cancer Cell 20: 487-499, 2011.

21. Westerheide SD, Anckar J, Stevens SM Jr, Sistonen L and Morimoto RI: Stress-inducible regulation of heat shock factor 1 by the deacetylase SIRT1. Science 323: 1063-1066, 2009.

22. Liu DJ, Hammer D, Komlos D, Chen KY, Firestein BL and Liu AY: SIRT1 knockdown promotes neural differentiation and attenuates the heat shock response. J Cell Physiol 229: 1224-1235, 2014.

23. Melhem H, Hansmannel F, Bressenot A, Battaglia-Hsu SF, Billioud V, Alberto JM, Gueant JL and Peyrin-Biroulet L: Methyl-deficient diet promotes colitis and SIRT1-mediated endoplasmic reticulum stress. Gut: Jan 20, 2015 (Epub ahead of print). doi: 10.1136/gutjnl-2014-307030.

24. Peck B, Chen CY, Ho KK, Di Fruscia P, Myatt SS, Coombes RC, Fuchter MJ, Hsiao CD and Lam EW: SIRT inhibitors induce cell death and p53 acetylation through targeting both SIRT1 and SIRT2. Mol Cancer Ther 9: 844-855, 2010. 
25. Karwaciak I, Gorzkiewicz M, Ryba K, Dastych J, Pulaski L and Ratajewski M: AC-93253 triggers the downregulation of melanoma progression markers and the inhibition of melanoma cell proliferation. Chem Biol Interact 236: 9-18, 2015.

26. Hoffmann G, Breitenbücher F, Schuler M and EhrenhoferMurray AE: A novel sirtuin 2 (SIRT2) inhibitor with p53-dependent pro-apoptotic activity in non-small cell lung cancer. J Biol Chem 21: 5208-5216, 2014.

27. Lee IH, Cao L, Mostoslavsky R, Lombard DB, Liu J, Bruns NE, Tsokos M, Alt FW and Finkel T: A role for the NAD-dependent deacetylase Sirtl in the regulation of autophagy. Proc Natl Acad Sci USA 105: 3374-3379, 2008.

28. Donmez G, Arun A, Chung CY, McLean PJ, Lindquist S and Guarente L: SIRT1 protects against $\alpha$-synuclein aggregation by activating molecular chaperones. J Neurosci 32: 124-132, 2012.

29. Björk JK and Sistonen L: Regulation of the members of the mammalian heat shock factor family. FEBS J 277: 4126-4139, 2010 .
30. Hietakangas V, Ahlskog JK, Jakobsson AM, Hellesuo M, Sahlberg NM, Holmberg CI, Mikhailov A, Palvimo JJ, Pirkkala L and Sistonen L: Phosphorylation of serine 303 is a prerequisite for the stress-inducible SUMO modification of heat shock factor 1. Mol Cell Biol 23: 2953-2968, 2003.

31. O'Callaghan-Sunol C and Sherman MY: Heat shock transcription factor (HSF1) plays a critical role in cell migration via maintaining MAP kinase signaling. Cell Cycle 5: 1431-1437, 2006.

32. Toma-Jonik A, Widlak W, Korfanty J, Cichon T, Smolarczyk R, Gogler-Piglowska A, Widlak P and Vydra N: Active heat shock transcription factor 1 supports migration of the melanoma cells via vinculin down-regulation. Cell Signal 27: 394-401, 2015.

33. Kwon SM, Kim SA, Yoon JH and Ahn SG: Transforming growth factor beta1-induced heat shock protein 27 activation promotes migration of mouse dental papilla-derived MDPC-23 cells. J Endod 36: 1332-1335, 2010. 